\title{
Fatal Ethylene Glycol Intoxication
}

\author{
A Orfanidis ${ }^{1}$, E Zaggelidou², N Raikos ${ }^{1}$, P Mylonakis ${ }^{2}$ and 0 Mastrogianni ${ }^{2 *}$ \\ ${ }^{1}$ Laboratory of Forensic Medicine Toxicology, Medical School, Aristotle University Thessaloniki, Greece \\ ${ }^{2}$ Laboratory of Forensic Service of Ministry of Justice, Transparency and Human Rights of Thessaloniki, Greece
}

Submission: February 17, 2017; Published: March 22, 2017

*Corresponding author: Orthodoxia Mastrogianni, Forensic Service of Thessaloniki, Palaia Symmahiki Odos, Diavata, 56334, Thessaloniki, Greece, Tel: 306981105262; +302310550413; Email: orthodoxiamastrogianni@hotmail.com

\begin{abstract}
Ethylene glycol (EG) can be found in many agents, such as antifreeze. Intoxications with EG occur infrequently, but may lead to fatalities if not recognized and treated promptly. Ethylene glycol presents great analytical challenge for forensic confirmation in postmortem toxicology. We describe fatal case of acute ethylene glycol poisoning following ingestion of anti-freezing agent and the development of simple, rapid and accurate GC/MS method for the detection and quantification of EG in biological fluids. It is the first fatal case, during the last 13 years, where cause of death was ascertained as EG intoxication at the Forensic Service of Thessaloniki, in Greece.
\end{abstract}

Keywords: Ethylene Glycol intoxication; Toxicological analysis; GC/MS

Abbreviations: EG: Ethylene Glycol; GC/MS: Gas chromatography-mass spectrometry; EI: Electron Impact

\section{Introduction}

Clinical and forensic laboratories frequently have to face cases of severe intoxication from ethylene glycol, because of its wide availability, and thus its use as an additive in various housekeeping and commercial products such as anti-freezing agent, car batteries and solvents for dyes. The majority of ethylene glycol poisonings is related to suicide attempts (intentional ingestion) and to misguided or debilitated alcoholics (accidental ingestion or cheap substitute of alcohol) [1]. Ethylene glycol is metabolized to several toxic organic acids by alcohol and aldehyde dehydrogenases [2]. While EG itself exerts an acute depression of the central nervous system, glycolate and other acidic metabolites seem to be responsible for the acute toxic effects such as acidosis, hyperventilation, hypoxia, congestive heart failure, and acute respiratory distress syndrome $[3,4]$. Oxalate causes delayed renal failure due to precipitation of calcium oxalate crystals. After 24 hours, renal symptoms typically predominate, which if left untreated can lead to multiorgan failure and finally death [5]. We present case report of fatal EG-ingestion and the development of new GC/MS method for the quantification of this analyte, in order to illustrate the lethal potential of this common and widely available product.

\section{Case Report}

Woman in her forties, with psychiatric history, presented to the emergency department of local hospital with mental status changes and clinical findings of acute drug intoxication, after previous suicide attempt with an anti-freezing agent and hypnotic pills. She died 24 hours after admission despite supportive care and treatment with sodium bicarbonate.

\section{Autopsy findings}

The autopsy revealed head injuries due to previous falling with no other significant macroscopic findings. The brain $(1450 \mathrm{~g})$ had no pathological or traumatic lesions with few atherosclerotic lesions. The heart (350 g) revealed atherosclerotic stenosis of LAD up to $50 \%$ and the lungs (left 600 g, right $620 \mathrm{~g}$ ) were edematous and congested. Multiple sections of the pulmonary parenchyma showed white foam indicating acute pulmonary edema. The kidneys (left $190 \mathrm{~g}$, right $180 \mathrm{~g}$ ) had no macroscopic findings. Liver (1440 g), spleen (170 g), pancreas (160 g) and adrenals had normal shape and texture upon dissection. The gastric mucosa was hemorrhagic with chemical gastritis erosions. Inside the stomach were found about $150 \mathrm{cc}$ of activated carbon, and few white remains of pharmaceutical pills. Cause of death was ascertained as poisoning.

\section{Materials and methods}

All chemicals used were of analytical reagent grade. Ethylene glycol was purchased from Merck (Darmstadt, Germany). Blood samples and gastric content were submitted to the laboratory analysis where they were stored at $-20^{\circ} \mathrm{C}$ before being analysed. 
All samples were screened by gas chromatography/mass spectrometry (GC/MS). Additionally, a rapid, simple and specific method for quantitative determination of EG in blood and other biological fluids (urine, gastric content) by GC/MS was developed in order to determine EG blood levels.

\section{Sample preparation}

Aliquots (200 l) of post-mortem biological fluids, along with matrix-matched calibrators and controls, were diluted with 200 acetonitrile and vortex mixed for $10 \mathrm{~min}$. After centrifugation at $12000 \mathrm{rpm}$ for $10 \mathrm{~min}$, of the supernatant was subjected to GC/ MS analysis.

\section{Gas chromatography-mass spectrometry}

GC/MS analysis was performed with Gas Chromatograph (Agilent Technologies) 7890A with MS 5975C inrtXL, EI/CI MSD with Triple-Axis detector. The mass spectrometer was operated with electron energy of $70 \mathrm{eV}$ in the electron impact (EI) mode. An Agilent J\&W HP-5ms fused-silica capillary column (30 0.25 mm i.d., 0.25 film thickness) was used for GC separation. GC oven temperature program was the following: $\min$ at $70^{\circ} \mathrm{C}$ and then from 70 to $100^{\circ} \mathrm{C}$ at $4^{\circ} \mathrm{C} / \mathrm{min}$. Solvent purging was used in splitless mode. The GC-MS interface was maintained at $300^{\circ} \mathrm{C}$. Helium was used as carrier gas at flow-rate of $0.46 \mathrm{~mL} / \mathrm{min}$. The acquisition mode was in full scan from 29 to $150 \mathrm{amu}$.

\section{Results and discussion}

Screening with GC/MS showed traces of alprazolam in woman's blood. Blood standards were calibrated between 100 $2000 \mathrm{mg} / \mathrm{L}$ ethylene glycol. The conditions of the present method succeeded in achieving linear relationships in the prementioned ranges (100-2000 $\mathrm{mg} / \mathrm{L})$ with coefficient of determination (R2 0.9982. Inter run precision was evaluated at EG concentrations of $100 \mathrm{mg} / \mathrm{L}$ (\%rsd: 14.48) and $1000 \mathrm{mg} / \mathrm{L}$ (\%rsd: 10.47), in six analytical runs. Recovery of EG in postmortem blood at concentrations $250 \mathrm{mg} / \mathrm{L}$ and $1250 \mathrm{mg} / \mathrm{L}$ was found to be $92 \%$ and $101 \%$ respectively (Table 1 ). After validation, the method was applied to the biological fluids of the descendant and EG was identified both in blood (chromatogram and mass spectrum are shown in Figures $1 \& 2$ respectively) and in gastric content. Quantitative result for postmortem blood was $408.4 \mathrm{mg} / \mathrm{L}$. Detection of EG in gastric content, in correlation with the high blood concentration, proves undoubtedly that the cause of death was the ingestion of the antifreeze. Adult patients are typically exposed when EG is ingested as cheap substitute for ethanol or in suicide attempts, in this case report we have described an example of the latter. There is lack of correlation between blood EG concentration and clinical symptoms because of rapid conversion of EG to its toxic metabolites [1].

Table 1: Linear and validation parameters of the in house GC/MS method for determination of EG.

\begin{tabular}{|c|c|c|c|c|c|c|}
\hline \multirow{2}{*}{ Compound } & \multirow{2}{*}{ Equation } & \multirow{2}{*}{$\mathbf{R}^{2}$} & \multicolumn{2}{|c|}{ Recovery (\%) } & \multicolumn{2}{|c|}{ Interrun precision (\%rsd) } \\
\cline { 3 - 6 } & & & $\mathbf{2 5 0} \mathbf{~ m g / L}$ & $\mathbf{1 2 5 0} \mathbf{~ m g / L}$ & $\mathbf{1 0 0} \mathbf{~ m g / L ~}$ & $\mathbf{1 0 0 0} \mathbf{~ m g / L}$ \\
\hline Ethylene & $9.9301 \mathrm{x}$ & 0.9982 & $92 \%$ & $101 \%$ & 14.48 & 10.47 \\
\hline Glycol & 4.2962 & & & & & \\
\hline
\end{tabular}

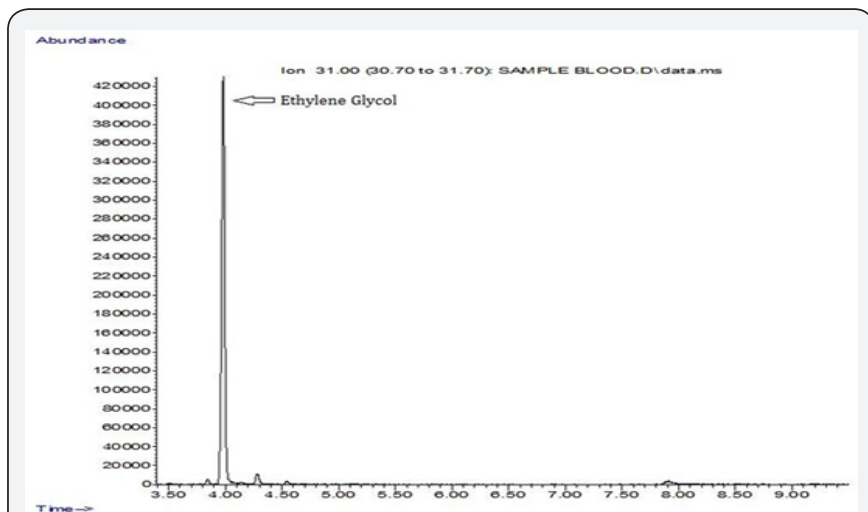

Figure 1: Extracted ion chromatogram for blood sample points out the existence of EG (characteristic ion $\mathrm{m} / \mathrm{z}: 31$ ).

The peak plasma concentration is reached approximately two hours after ingestion and the half life of EG is about 3-7h [6]. Additional to the toxicological results, histopathological analysis revealed that the kidneys showed diffuse tubular epithelium and multiple translucent crystal depositions, which exhibited radial and shell-like patterns. Early therapy consists of correction of the acidosis; latter treatment is concerned with the careful management of renal failure [7]. In order to face this

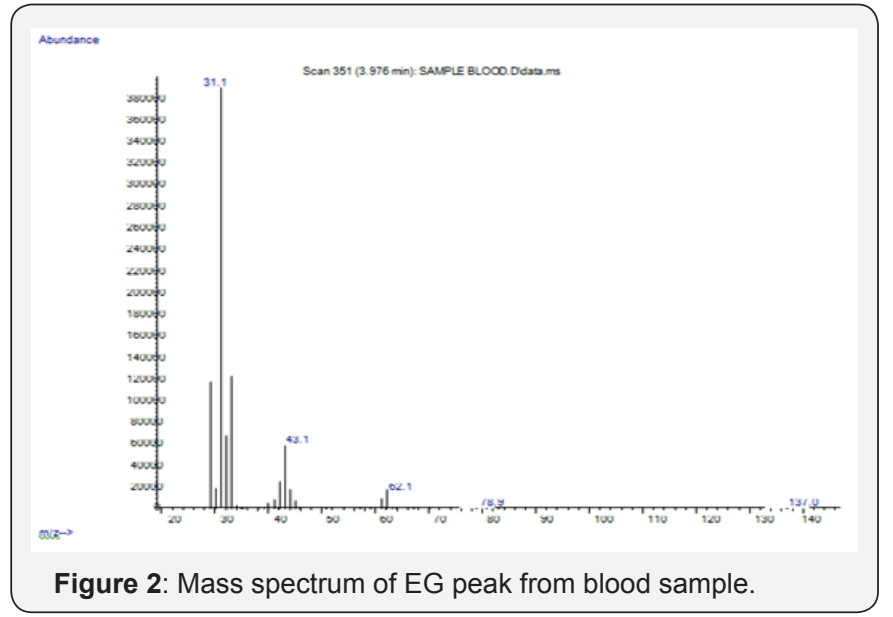

fatal case in our laboratory, we developed rapid and specific GC/ MS method with satisfactory reproducibility, linearity, precision and recovery, which represents useful analytical tool for rapid and specific diagnosis of EG poisoning and for monitoring of its treatment. To our knowledge, it is the first described case of fatal EG intoxication in Northern Greece during the last 10 years. Children may be exposed by accidental ingestion caused by decantation of EG to unlabeled bottles, so it is of great importance 
the proper labeling of antifreeze containers. The EG container should be stored separately from food and should have child proof cap. The forensic examiner should investigate these cases with special emphasis on prevention, and should communicate the experiences thus gained to the proper authorities in order to increase public safety.

\section{References}

1. Leth PM, Gregersen M (2004) Ethylene glycol poisoning. Forensic Sci Int 155(2-3): 179-184

2. Cruzan G, Corley RA, Hard GC, Mertens JJ, McMartin KE, et al. (2004) Subclinical Toxicity of ethylene glycol in Wistar and F344 rats related to metabolism and clearance of metabolites. Toxicol Sci 81(2): 502511
3. Keith L Clay, Murphy C Robert (1977) On the metabolic acidosis of ethylene glycol intoxication, Toxicology and Applied Pharmacology 39(1): 39-49.

4. Jacobsen D, McMartin KE (1986) Methanol and ethylene glycol poisonings. Mechanism of toxicity, clinical course, diagnosis and treatment. Med Toxicol 1(5): 309-343.

5. Bove KE (1966) Ethylene glycol toxicity, Am J Clin Pathol 45(1): 46-50.

6. Jacobsen D, Hewlett TP, Webb R, Brown ST, Ordinario AT, et al. (1988) Ethylene glycol intoxication:Evaluation of kinetics and cryastalluria, Am J Med 84(1): 145-152.

7. Wiley JF (1999) Novel therapies for ethylene glycol intoxication. Curr Opin Pediatr (11): 269-273.

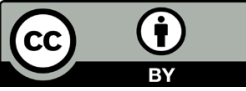

This work is licensed under Creative Commons Attribution 4.0 License

DOI: $10.19080 /$ JFSCI.2017.02.555589

\section{Your next submission with Juniper Publishers will reach you the below assets}

- Quality Editorial service

- Swift Peer Review

- Reprints availability

- E-prints Service

- Manuscript Podcast for convenient understanding

- Global attainment for your research

- Manuscript accessibility in different formats

(Pdf, E-pub, Full Text, Audio)

- Unceasing customer service

Track the below URL for one-step submission https://juniperpublishers.com/online-submission.php 